\title{
PROGRESS TOWARDS A CONJECTURE ON THE MEAN-VALUE OF TITCHMARSH SERIES-II
}

\author{
By K. RAMACHANDRA
}

\section{Introduction}

The result of this paper may be considered as complementary to that of my earlier paper [2], on Titchmarsh series. Although not as interesting as the earlier result, the result of the present paper finds a nice application, (See [1]). In [3] I defined a class of series called Titchmarsh series and $I$ now start by recalling its definition.

Titchmarsh Series. (or briefly K D T series).

Let $A>10$ be a constant.

$$
\begin{aligned}
& \text { Let } \frac{1}{\mathrm{~A}}<\mu_{1}<\mu_{2}<\ldots \text { where } \\
& \frac{1}{\mathrm{~A}}<\mu_{n+1}-\mu_{n}<\mathrm{A}(\text { for } n=1,2,3, \ldots) \text { ). }
\end{aligned}
$$

(In [2] the notation is slightly different and we have used there $\lambda_{n}$ instead of $\boldsymbol{\mu}_{n}$ and for simplicity assumed $\lambda_{1}=1$. Also we have used there $a_{1}, a_{2}, \ldots$ in place of our present $b_{1}, b_{2} \ldots$ and assumed for simplicity $a_{1}=1$. We have written there $\mathrm{F}(s)$ instead of $\mathrm{F}_{0}(s)$ ). Let $b_{1}, b_{2}, \ldots$ be a sequence of complex numbers possibly depending on a parameter $H>10$ such that $\left|b_{n}\right|<\left(\mu_{n} \mathrm{H}\right)$ A . Put $F_{0}(s)=\sum_{n=1}^{\infty} b_{n} \mu_{n}^{-s}$ where $s=\sigma+$ it. 
$\mathbf{F}_{0}(s)$ is called a KDT series if there exists a constant $A>10$ and a system of rectangles $R(T, T+H)$ defined by $\{\sigma>0$, $\mathrm{T}<t<\mathrm{T}+\mathrm{H}\}$ where $10<\mathrm{H}<\mathrm{T}$, and $\mathrm{T}$ (which may be related to $H$ ) tends to infinity, and $F_{0}(s)$ admits an analytic continuation into these rectangles and the maximum of $\left|F_{0}(s)\right|$ taken over $R(T, T+H)$ does not exceed $\operatorname{Exp}\left(H^{A}\right)$.

I then made the following coajecture

\section{Conjecture}

For a KDT series $\mathrm{F}_{0}(s)$, we have,

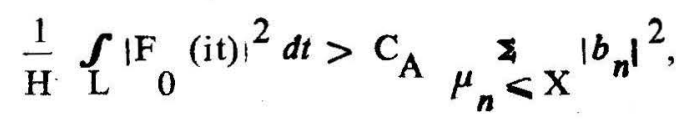

where $\mathrm{X}=2+\mathrm{D}_{\mathrm{A}} \mathrm{H}, \mathrm{L}$ denotes the side $(\sigma=0, \mathrm{~T}<t<\mathrm{T}+\mathrm{H})$ of $\mathrm{R}(\mathrm{T}, \mathrm{T}+\mathrm{H})$, and $\mathrm{C}_{\mathrm{A}}$ and $\mathrm{D}_{\mathrm{A}}$ are positive constants depending only on $\mathrm{A}$, provided $\mu_{1}=b_{1}=1$.

I proved the following theorem.

\section{Theorem 1}

Under the restrictions $\mu_{1}=b_{1}=1$, we huve,

$$
\frac{\frac{1}{\mathrm{H}} \int_{\mathrm{L}}\left|\mathrm{F}_{0}(\mathrm{it})\right|^{2} d t>\mathrm{C}_{\mathrm{A}} \boldsymbol{\mu}_{n}^{\mathrm{\Sigma}}<\mathrm{X}{ }_{n}^{|\boldsymbol{b}|^{2}}}{\left(1-\frac{\log \mu n}{\log \mathrm{H}}+\frac{1}{\log \log \mathrm{H}}\right),}
$$

where $\mathrm{X}=2+\mathrm{D}_{\mathrm{A}} \mathrm{H}$ and, $\mathrm{C}_{\mathrm{A}}$ and $\mathrm{D}_{\mathrm{A}}$ are effective positive constants depending only on $\mathrm{A}$.

I now prove the following theorem. 


\section{Theorem 2}

For some convenience let us assume in the definition of Titchmarsh series $\mathrm{F}_{0}(s)$ the rectangles $\mathrm{R}(\mathrm{T}, \mathrm{T}+\mathrm{H})$ to be $(\sigma>\beta, T<t<\mathrm{T}+\mathrm{H})$ where $\beta$ is a positive constant such that $0<\beta<\alpha_{1}<\frac{1}{2}$, where $\alpha_{1}$ is another constant. Let $k \geq 2$ be an integer and write $\mathrm{F}(s)=\left(\mathrm{F}_{0}(s)\right)^{k}=$ $\sum_{n=1}^{\infty}\left(a_{n} \lambda_{n}^{-s}\right)$, a series which is surely convergent where $\mathrm{F}_{0}(s)$ is absolutely convergent. Put $\mathrm{Y}=(\mathrm{M}+\mathrm{H})^{\lambda}$ where $\lambda=k \mathrm{~A}^{100}\left(\alpha_{1}-\beta\right)^{-10}$ and $\mathrm{M}=$ maximum of $\left|\mathrm{F}_{0}(s)\right|$ taken over $\mathrm{R}(\mathrm{T}, \mathrm{T}+\mathrm{H})$. Define the entire function $\phi(s)$ by

$$
\phi(s)=\sum_{n=1}^{\infty} a_{n} \lambda_{n}^{-s} \Delta\left(\frac{Y}{\lambda_{n}}\right)
$$

where for $\mathrm{X}>0, \Delta(\mathrm{X})$ is defined by

$$
\Delta(\mathrm{X})=\frac{1}{2 \pi i} \int_{2}^{2+i \infty} \mathrm{X}^{\mathrm{W}} \operatorname{Exp}\left(\mathrm{W}^{4 a+2}\right) \frac{d \mathrm{~W}}{\mathrm{~W}} .
$$

a being a suitable positive integer constant at our choice. We now suppose that $\alpha, \beta, \alpha_{1}, \alpha_{2}, \alpha_{3}$ are constants satisfying

$$
\alpha<\beta<\alpha_{1}<\alpha_{2}<\alpha_{3}<\alpha+\frac{1}{2} \text {. }
$$

Put $\mathrm{X}-\left[2^{-\mathrm{A}-100} \mathrm{H}\right]+2$ and

$\mathrm{V}(\sigma)=\frac{1}{\mathrm{H}} \sum_{\mu_{n}<\mathrm{X}}\left|b_{n}\right|^{2}\left(\frac{\mathrm{H}}{\mu_{n}}\right)^{2 \sigma}$. 
Then we have,

$$
\frac{1}{\mathrm{H}} \int_{\mathrm{L}}|\phi(\alpha+i t)|^{2 k^{-1}} d t
$$

$$
\gg V(d) H^{1-2 \alpha}\left\{\frac{V\left(\alpha_{1}\right)}{V\left(\alpha_{3}\right)}\right\}^{\frac{\alpha_{2}-\alpha}{\alpha_{3}-\alpha_{2}}}
$$

provide tonly that $\mathrm{V}(\alpha) \neq 0$ and $(\log \mathrm{V}(\alpha))(\log \mathrm{H})^{-1}$ is bounded below by a negative constant. Here the constant implied by the Vinogradov symbol $\gg$ depends only on $A, k, \alpha, \beta, \alpha_{1}$, $\alpha_{2}, \alpha_{3}$ and the negative constant referred to just now. Further it is effective.

Remark: It will be clear from the proof that if $\mu_{n}=n(n=1,2,3, \ldots)$ then we can choose $\mathrm{X}=\left[\frac{\mathrm{H}}{100}\right]+2$.

The object of this paper is to prove theorem 2. The proof of the theorem is fairly long. The proof depends upon a special case of a convexity theorem of R. M. Gabriel which we state below (in the notation of D. R. Heath-Brown's paper [2] ; for the more general theorem of Gabriel see the reference in [2] or Titchmarsh's famous book [5] pages 203 and 337.)

\section{Theorem 3}

Let $f(z)$ be regular in the infinite strip $\alpha<\operatorname{Re} z<\beta$ and continuous for $\alpha<\operatorname{Re} z \leqslant \beta$. Suppose $f(z) \rightarrow 0$ as $|\operatorname{Im} z| \rightarrow \infty$, uniformly in $\alpha<\operatorname{Re} z<\beta$. Then for $\alpha \leqslant \gamma \leqslant \beta$, and any $q>0$, we have 


$$
\begin{aligned}
\int_{-\infty}^{\infty}|f(\gamma+i t)|^{q} d t< & \frac{\left(\int_{-\infty}^{\infty}|f(\alpha+i t)|^{q} d t\right)^{\frac{\beta-\gamma}{\beta-\alpha}}}{\left.C_{-\infty}^{\infty}|f(\beta+i t)|^{q} d t\right)^{\frac{\gamma-\alpha}{\beta-\alpha}}}
\end{aligned}
$$

provided the right hand side is finite.

Apart from this we have to use a well-known theorem of Montgomery and Vaughan. For reference see for instance my paper [4], where 1 give a simple proof of a weaker result which is sufficient for the purposes of this paper.

We now split up the proof of theorem 2 into several steps and give a brief sketch of these steps.

\section{Proof of Theorem 2}

Step I. Let

$$
\begin{aligned}
I(\sigma)=\frac{1}{H} \int_{T}^{T+H} & \left(\int_{-\infty}^{\infty}|\phi(s)|^{2 / k}\right. \\
& \left.\left|\operatorname{Exp}\left(\left(s-i t_{0}\right)^{4 a+2}\right)\right| d t\right) d t_{0} .
\end{aligned}
$$

and assume that $\mathrm{I}(\alpha)<\mathrm{V}(\alpha) \mathrm{H}^{1-2 \alpha}$. The constant $a$ shall be a sufficiently large positive integer. As already stated we set

$$
\begin{aligned}
\mathrm{X} & =\left[^{-\mathrm{A}-100} \mathrm{H}\right]+2 \text {, and } \\
\mathrm{V}(\sigma) & =\frac{1}{\mathrm{H}} \sum_{\mu_{n}<\mathrm{X}}\left|b_{n}\right|^{2}\left(\frac{\mathrm{H}}{\mu_{n}}\right)^{2 \sigma} \text { and }
\end{aligned}
$$

we impose $\alpha<\beta<\alpha_{1}<\alpha_{2}<\alpha_{3}<\alpha+\frac{1}{2}$. 
Step 2. Next we write

$$
\begin{aligned}
\mathrm{J}(\sigma)=\frac{1}{\mathrm{H}} \int_{\mathrm{T}}^{\mathrm{T}+\mathrm{H}} & \left(\int_{-\infty}^{\infty}\left|\phi(s)-\mathrm{P}^{\mathrm{k}}(s)\right|^{2 / k}\right. \\
& \left.\left|\operatorname{Exp}\left(\left(s-i t_{0}\right)^{4 a+2}\right)\right| d t\right) d t_{0}
\end{aligned}
$$

where $\mathrm{P}(s)={ }_{\mu_{n}}{ }_{n}<\mathrm{X}{ }_{n}{ }_{n}{ }_{n}^{-s}$. It is easily seen that $\Delta(\mathrm{X})=O\left(\mathrm{X}^{\mathrm{B}}\right)$ and also $1+O\left(\mathrm{X}^{-\mathrm{B}}\right)$ where $\mathrm{B}>0$ is an arbitrary constant and the $O$-constant depends only on $\mathrm{B}$ and $\boldsymbol{a}$. Again $a_{n}=\mu_{\mu_{1}} \ldots \mu_{n_{k}}=\lambda_{n}\left(b_{n_{1}} b_{n_{2}} \ldots b_{n_{k}}\right)$ and for all $\mathrm{N}>1$, we have $\mathrm{N}<\mu_{n_{1}} \sum_{\mu_{n_{k}}<2 \mathrm{~N}}=O\left(\mathrm{~N}^{k}\right)$. From these remarks it is clear that $\phi(s)-\mathrm{P}^{k}(s)$ decays fast enough to ensure $J(\sigma)<1$ when $\sigma$ is large enough. Now from an easy application of a theorem of Gabriel (Theorem 3 above) it follows that in $\sigma>\alpha, \mathrm{J}(\sigma)$ is $\ll_{\varepsilon}[\mathrm{J}(\alpha)]^{1-\varepsilon}$ for every positive constant $\varepsilon$ uniformly in $\sigma$, and so in $(\sigma>\beta$, $\mathrm{T}<t \leqslant \mathrm{~T}+\mathrm{H}), \quad|\phi(s)|$ is bounded above by a constant power of $\mathrm{H}$. (Here for getting the last bound we have to use the fact that for any analytic function $\phi(s), \mid \phi(s) i^{2 / k}$ is bounded by its mean value over a disc of (positive but sufficiently small) constant radius with $s$ as centre).

Step 3. An easy application of a well-known MontgomeryVaughan theorem (refer [4] for instance) shows that

$$
\begin{array}{r}
\frac{1}{\mathrm{H}} \int_{\mathrm{T}}^{\mathrm{T}+\mathrm{H}}\left(\int_{-\infty}^{\infty}|\mathrm{P}(s)|^{2}\left|\operatorname{Exp}\left[\left(s-i t_{0}\right)^{4 a+2}\right]\right| d t\right) d t_{0} \\
=O\left(\mathrm{~V}(\sigma) \mathrm{H}^{1-2 \sigma}\right) .
\end{array}
$$


From this and the estimate $J(\sigma) \ll_{\varepsilon}(J(\alpha))^{1-\varepsilon}$ it follows that

$$
\begin{gathered}
\int_{\alpha}^{\mathrm{A}^{3}}\left(\frac { 1 } { \mathrm { H } } \int _ { \mathrm { T } } ^ { \mathrm { T } + \mathrm { H } } \left(\int _ { - \infty } ^ { \infty } \left(\left|\phi(s)-\mathrm{P}^{k}(s)\right|^{2 / k}\right.\right.\right. \\
\left.\left.\left.+|\mathrm{P}(s)|^{2}\right)\left|\operatorname{Exp}\left(\left(s-i t_{0}\right)^{4 a+2}\right)\right| d t\right) d t_{0}\right) d \sigma \\
=O\left((\mathrm{~J}(\alpha))^{1-\varepsilon}+\mathrm{V}(\alpha) \mathrm{H}^{1-2 \alpha}\right) .
\end{gathered}
$$

Note that $\mathrm{V}(\sigma)$ and $\mathrm{V}^{*}(\sigma) \mathrm{H}^{1-2 \sigma}$ are respectively monotonic increasing and monotonic decreasing functions of $\sigma$, where $\mathrm{V}^{*}(\sigma)$ is the same as $\mathrm{V}(\sigma)$ with the terms $\mu_{n}<1$ omitted. From now on we assume that $\mathrm{V}(\alpha)$ is bounded below by a constant negative power of $H$. Under this assumption it follows that the integral just considered is

$$
o_{\varepsilon}\left(\mathrm{V}(\alpha) \mathrm{H}^{1-2 \alpha+\varepsilon}\right) \text { for every positive constant } \varepsilon \text {. }
$$

Hence there exist intervals $I_{1}$ and $I_{2}$ contained in $\left(T, T+\frac{H}{10}\right)$ and $\left(T+H-\frac{H}{10}, T+H\right)$ respectively, for which the lengths are $4 \mathrm{H}^{\varepsilon} \quad(\delta$ being any fixed constant satisfying $\left.0<\delta<\frac{1}{100}\right)$ each, and further $I\left(I_{1}, \alpha\right)$

$$
\begin{aligned}
=\int_{\alpha}\left(\frac { 1 } { \mathrm { H } } \int _ { \mathrm { I } _ { 1 } } \int _ { - \infty } ^ { \infty } \left(\left|\phi(s)-\mathrm{P}^{k}(s)\right|^{2 / k}\right.\right. \\
\left.\left.\quad+|\mathrm{P}(s)|^{2}\right)\left|\operatorname{Exp}\left(\left(s-i t_{0}\right)^{4 a+2}\right)\right| d t d t_{0}\right) d \sigma
\end{aligned}
$$

and $l\left(I_{2}, \alpha\right)$ defined similarly (by replacing $I_{1}$ by $I_{2}$ ) satisfy

$$
\mathrm{I}\left(\mathrm{I}_{1}, \alpha\right)+\mathrm{I}\left(\mathrm{I}_{2}, \alpha\right)=O\left(\mathrm{~V}(\alpha) \mathrm{H}^{1-2 \alpha+\varepsilon}\right)
$$


Hence by the principle for the mean value over discs referred to in the second step, we see that in $\left(B \leq \sigma \leq A^{2}\right.$, $t$ in any of the intervals $I_{1}, I_{2}$ ) we have

$$
|\phi(s)|^{2 / k}=O\left(\mathrm{~V}(\alpha) \mathrm{H}^{-2 \alpha+2 \varepsilon+\delta}\right) \text {. }
$$

Step 4. Let $H_{1}$ and $H_{2}$ be the mid points of $I_{1}$ and $I_{2}$ respectively. We now obtain a lower bound for at least one of the mean-values $K\left(\alpha_{1}\right)$ or $K\left(\alpha_{2}\right)$ where $K(\sigma)$ is defined by

$$
K(\sigma)=\frac{1}{\mathrm{H}_{2}-\mathrm{H}_{1}} \int_{\mathrm{H}_{1}}^{\mathrm{H}_{2}}\left|\mathrm{~F}_{0}(s)\right|^{2} d t,\left(\dot{\beta}<\sigma<\mathrm{A}^{2}\right) .
$$

Note that when we replace $H_{1}$ and $H_{2}$ by other points in $I_{1}$ and $I_{2}$ the mean value $K(\sigma)$ changes by an amount which is at most $O(E)$ where $E=V(\alpha) H^{-2 \alpha+2 \varepsilon+28}$. Hence if $\sigma$ denotes any of $\alpha_{2}$ or $\alpha_{3}$ and $\mathrm{H}_{1}<t<\mathrm{H}_{2}$, we see that if $j$ is a large positive integer constant,

(1) $\begin{aligned} \frac{j !}{2 \pi i} \int_{\mathrm{L}_{0}} \frac{\mathrm{F}_{0}(s+w)(2 \mathrm{X})^{w}}{w(w+1) \ldots(w+j)} d w \\ =\sum_{\mu_{n} \leqslant 2 \mathrm{X}} b_{n} \mu_{n}^{-s}\left(1-\frac{\mu_{n}}{2 \mathrm{X}}\right)^{j},\end{aligned}$

where $\mathrm{L}_{0}$ is the line $\operatorname{Re} w=A^{2}$. Deform the line $\mathrm{L}_{0}$ to the contour described by the lines $L_{1}, L_{2}, L_{3}, L_{4}, L_{5}$ ( in this order) defined as follows. Let $H_{3}=H_{1}-H^{\delta}$, $\mathrm{H}_{4}=\mathrm{H}_{2}+\mathrm{H}^{\delta}$ where $\delta$ is a small positive constant. $\mathrm{L}_{1}$ and 
$\mathrm{L}_{5}$ are the portions $\mathrm{Im} w-\mathrm{H}_{3}$ and $\operatorname{Im} w \geqq \mathrm{H}_{4}$ respectively of $\mathrm{L}_{0} \cdot \mathrm{L}_{2}$ is the line segment

$\left(\operatorname{Im} w=-\mathrm{H}_{3}, \alpha_{1}-\sigma \leqslant \operatorname{Re} w \leqslant \mathrm{~A}^{2}\right)$

and $\mathrm{L}_{4}$ is the line segment

$\left(\operatorname{Im} w=\mathrm{H}_{4}, \alpha_{1}-\sigma<\operatorname{Re} w \leqslant \mathrm{~A}^{2}\right)$.

$\mathrm{L}_{3}$ is the line segment $\left(\operatorname{Re} w=\alpha_{1}-\sigma,-\mathrm{H}_{3}<\operatorname{Im} w<\mathrm{H}_{4}\right)$. Taking the mean square after deformation of $\mathrm{L}_{0}$ we find from the equation (1), (Note that the only pole to be taken care of is $w=0$ ),

(2)

$$
\left\{\begin{array}{r}
\mathrm{K}(\sigma) \ll \mathrm{V}_{1}(\sigma) \mathrm{H}^{1-2 \sigma}+\left(\mathrm{K}\left(\alpha_{1}\right)+\mathrm{E}\right) \mathrm{H}^{-2\left(\sigma-\alpha_{1}\right)} \\
\quad \text { (where } \mathrm{V}_{1}(\sigma) \text { is defined below) and also } \\
\mathrm{V}(\sigma) \mathrm{H}^{1-2 \sigma} \ll \mathrm{K}(\sigma)+\left(\mathrm{K}\left(\alpha_{1}\right)+\mathrm{E}\right) \mathrm{H}^{-2\left(\sigma-\alpha_{1}\right)}
\end{array}\right.
$$

The reason for this is that the mean square of the RHS of $(1)$ is $\gg$ and $\ll \mathrm{V}_{1}(\sigma) \mathrm{H}^{1-2 \sigma}$ where $\mathrm{V}_{1}(\sigma)$ is defined by $\mathrm{V}_{1}(\sigma)=\left.\frac{1}{\mathrm{H}} \sum_{\mu_{n} \leqslant \mathrm{X}}|b|_{n}\right|^{2}\left(1-\frac{\mu_{n}}{2 \mathrm{X}}\right)^{2 \mathrm{j}}\left(\frac{\mathrm{H}}{\mu_{n}}\right)^{2 \sigma}$.

Since $V(\alpha) \leqslant V(\sigma)$ we may omit the term containing $E$ in the second of the equations (2), provided $\alpha_{1}-\alpha<1$ (which is true because of our assumptions). This gives us $V(\sigma) H^{1-2 \sigma} \ll K(\sigma)+K\left(\alpha_{1}\right) H^{-2\left(\sigma-\alpha_{1}\right)} ;\left(\sigma=\alpha_{2}, \alpha_{3}\right)$

If we put $\sigma=\alpha_{2}$ we get a lower bound for one at least of the quantities $K\left(\alpha_{1}\right)$ or $K\left(\alpha_{2}\right)$.

We now deduce from the last inequality

$$
\begin{gathered}
\mathrm{V}(\sigma) \mathrm{H}^{1-2 \sigma} \ll \mathrm{I}(\sigma)+\mathrm{I}\left(\alpha_{1}\right) \mathrm{H}-2\left(\sigma-\alpha_{1}\right), \\
\left(\sigma=\alpha \frac{k}{2}, \alpha_{3}\right) .
\end{gathered}
$$


This is possible since in the range $\left(\sigma>\alpha_{1}, \mathrm{H}_{3}<t<\mathrm{H}_{4}\right)$, $\left|\mathrm{F}_{0}(s)\right|^{2}$ is a very good approximation (in the mean) to $\mid \phi(s) i^{2 / k}$ and we leave the details as an exercise. From the last inequality it follows that $I(\sigma) \gg V(\sigma) \mathrm{H}^{1-2 \sigma}$, for one at least of the values $\sigma=\alpha_{1}$ or $\alpha_{2}$.

Next by the convexity theorem of Gabriel (Theorem 3), we find that with the value of $\sigma\left(\alpha_{1}\right.$ or $\alpha_{2}$ determined),
(3) $(\mathrm{I}(\sigma))^{\alpha_{3}-\alpha}<(\mathrm{I}(\alpha))^{\alpha_{3}-\sigma}\left(\mathrm{I}\left(\alpha_{3}\right)\right)^{\sigma-\alpha}$.

Moreover by the arguments used in the first of the inequalities in (2) we get (by taking $X$ in place of $2 X$ in (1)).

$$
\begin{aligned}
& \mathrm{I}\left(\alpha_{3}\right) \ll V\left(\alpha_{3}\right) \mathrm{H}^{1-2 \alpha_{3}}+(\mathrm{I}(\sigma)+\mathrm{E}) \mathrm{H}^{-2\left(\alpha_{3}-\sigma\right)}+\mathrm{E} . \\
& \text { Now } \mathrm{E}=\mathrm{V}(\alpha) \mathrm{H}^{-2 \alpha+2 \varepsilon+2<}<\mathrm{V}\left(\alpha_{3}\right) \mathrm{H}^{1-2 \alpha_{3}}
\end{aligned}
$$

(since by our assumptions, $\alpha_{3}-\alpha<\frac{1}{2}$ ) by a small choice of the positive constants $\varepsilon, \gamma$. Thus we get

$$
I\left(\alpha_{3}\right) \ll V\left(\alpha_{3}\right) H^{1-2 \alpha_{3}}+I(\sigma) H^{-2\left(\alpha_{3}-\sigma\right)},
$$

and so by (3)

(4) $(\mathrm{I}(\sigma))^{\alpha_{3}-\alpha}$

$\ll(\mathrm{I}(\alpha))^{\alpha_{3}-\sigma}\left(\mathrm{V}\left(\alpha_{3}\right) \mathrm{H}^{1-2 \alpha_{3}}+\mathrm{I}(\sigma) \mathrm{H}^{-2\left(\alpha_{3}-\sigma\right)}\right)^{\sigma-\alpha}$.

This holds for either $\sigma=\alpha_{1}$ or $\sigma=\alpha_{2}$ and gives us 
$\int$ Either $\mathrm{I}(\alpha) \gg \mathrm{V}(\sigma) \mathrm{H}^{1-2 \alpha}>\mathrm{V}(\alpha) \mathrm{H}^{1-2 \alpha}$,

(5)

$$
\begin{array}{r}
\text { or }(\mathrm{I}(\alpha))^{\alpha_{3}-\sigma} \gg\left(\mathrm{V}(\sigma) \mathrm{H}^{1-2 \sigma}\right)^{\alpha_{3}-\alpha} \\
\left(\mathrm{V}\left(\alpha_{3}\right) \mathrm{H}^{1-2 \alpha_{3}}\right)^{\alpha-\sigma} .
\end{array}
$$

The second of these inequalities gives

$$
I(\alpha) \gg H^{1-2 \alpha} V(\sigma)\left\{\frac{V(\sigma)}{V\left(\alpha_{3}\right)}\right\}^{\frac{\sigma-\alpha}{\alpha_{3}-\sigma}}
$$

Since $\mathrm{V}(\alpha)<\mathrm{V}\left(\alpha_{1}\right) \leqslant \mathrm{V}(\sigma) \leq \mathrm{V}\left(\alpha_{3}\right)$ and since $\frac{u-\alpha}{\alpha_{3}-u}$

is an increasing function of $u$ in $\beta \leqslant u<\alpha_{3}$, we get finally

$$
I(\alpha) \gg V(\alpha) H^{1-2: x}\left\{\frac{V\left(\alpha_{1}\right)}{V\left(\alpha_{3}\right)}\right\}^{\frac{\alpha_{2}-\alpha}{\alpha_{3}-\alpha_{2}}}
$$

Step 5. Step 4 nearly completes the proof. For we could have started wi th a slight modification of $I(\sigma)$ by averaging over a slightly smaller interval contained in $(T, T+H)$ instead of $(T, T+H)$. For instance by cutting off bits of length $\mathrm{H}^{\delta}$ on either side. The decaying factor $\operatorname{Exp}\left(\left(s-i t_{0}\right)^{4 a+2}\right)$ enables us to replace the modified

$$
\text { I }(\sigma) \text { by } \frac{1}{\mathrm{H}} \int_{\mathrm{T}}^{\mathrm{T}+\mathrm{H}}|\phi(s)|^{2 / k} d t
$$

in the last lower bound.

Steps 1, 2, 3, 4 and 5 complete the proof of theorem 2 . 


\section{Theorem 4}

With the notation of theorem 2 , we have,

$$
\begin{aligned}
\frac{1}{H} \int_{T}^{T+H}|\phi(\alpha+i t)|^{2} d t \gg \\
\left(V(\alpha) H^{1-2 \alpha}\left\{\frac{V\left(\alpha_{1}\right)}{V\left(\alpha_{3}\right)}\right\}^{\frac{\alpha_{2}-\alpha}{\alpha_{3}-\alpha_{2}}}\right)^{k}
\end{aligned}
$$

where the constant implied by the Vinogradov symbol is effective Remark. This theorem will be used in [1].

\section{References}

I. R. Balasubramanian And K. Ramachandra, Some problems of analytic number theory-III, HardyRamanujan Journal, Vol. 4 (1981).

2. D. R. Heath-Brown, Fractional moments of the Riemann zeta-function, (to appear).

3. K. Ramachandra, Progress towards a conjecture on the mean-value of Titchmarsh Series, Proceedings of the Durham Conference on Analitic number theory, (July-August 1979), (to appear).

4. K. Ramachand,a Some remarks on a theorem of Montgomery and Vaughan, J. of Number theory, Vol. 11 (1975), 465-47I.

5. E. C. Ticchmarsh, The theory of the Riemann zetafunction, Oxford (1951).

Manuscript Completed In The Final Form On 11-Oct-1980

School of Mathematics

Tata Institute of Fundamental Research

Homi Bhabha Road

Colaba, Bombay 400005

(India). 